\title{
Acute interstitial nephritis during rifampicin therapy can be a paradoxical response: a case report
} Jan van der Meulen $^{1 *}$, Gijs MT de Jong ${ }^{2}$ and Pieter J Westenend ${ }^{3}$

Address: ${ }^{1}$ DIANET, Utrecht, The Netherlands, ${ }^{2}$ Department of Nephrology, Albert Schweitzer Hospital, Dordrecht, The Netherlands and ${ }^{3}$ Laboratory for Pathology, Dordrecht, The Netherlands

Email: JM* - debeteredokter@gmail.com; GJ - G.M.T.deJong@asz.nl; PW - PWestenend@paldordt.nl

* Corresponding author

Published: 3 April 2009

Received: 12 January 2009

Cases Journal 2009, 2:6643 10.1186/1757-1626-2-6643

Accepted: 17 February 2009

This article is available from: http://casesjournal.com/casesjournal/article/view/2/4/6643

(C) 2009 der Meulen et al; licensee Cases Network Ltd.

This is an Open Access article distributed under the terms of the Creative Commons Attribution License (http://creativecommons.org/licenses/by/3.0),

which permits unrestricted use, distribution, and reproduction in any medium, provided the original work is properly cited.

\begin{abstract}
An 18-year-old Ethiopian woman presented with debilitating back pain and high fever. X-ray examinations showed diffuse pulmonary tuberculosis and a psoas abscess. After starting rifampicin, isoniazid, ethambutol and pyrazinamide, acute interstitial nephritis developed that spontaneously recovered. According to Harrison's Online rifampicin should have been causative, but the spontaneous recovery excluded that possibility. The clinical course fit the diagnosis of a paradoxical response, for which recently risk factors have been described. Thus, a paradoxical response should be added to the list of causes of interstitial nephritis in tuberculosis patients and in such cases rifampicin could be continued.
\end{abstract}

\section{Introduction}

Rifampicin, mostly in combination with isoniazid, ethambutol and pyrazinamide, is the first-line therapy for tuberculosis. Rifampicin's most feared side effect is hepatotoxicity. However, nephrotoxicity such as acute tubular necrosis and interstitial nephritis (IN) have also been reported. In case of acute tubular necrosis, rifampicin-dependent antibodies have been found, suggesting a causal relationship between rifampicin and renal failure [1]. In acute IN only a temporal relationship is reported $[2,4]$. In spite of the unproven causal relation, Harrison's online lists rifampicin as cause of IN [5]. We present a patient with miliary tuberculosis, who developed IN during rifampicin, yet spontaneously recovered. Our observation and recent literature suggest that in case of hematogenously disseminated tuberculosis, a paradoxical response (PR) should be added to the list of possible causes of IN.

\section{Case presentation}

An 18-year-old Ethiopian woman was presented with debilitating back pain, weight loss and high fever. A month earlier an abscess of her right buttock was surgically drained. Physical examination revealed an ill-looking young woman with a temperature of $40.1^{\circ} \mathrm{C}$. The abdomen was painful at palpation and the liver slightly enlarged. The chest X-ray was compatible with diffuse pulmonary tuberculosis. A CT scan of the abdomen showed a psoas abscess on the left side. Laboratory data revealed: CRP $191 \mathrm{mg} / \mathrm{l}, \mathrm{Hb} 6.0 \mathrm{mmol} / \mathrm{l}$, lymphocyte 
count $0.8 \times 10^{9} / \mathrm{l}$, creatinine $93 \mathrm{micromol} / \mathrm{l}$, blood urea nitrogen $3.8 \mathrm{mmol} / \mathrm{l}$ and serum albumin $20 \mathrm{~g} / \mathrm{l}$. Urinalysis showed no protein, cells or casts. HIV serology was negative. Acid-fast bacilli were found in the pus of the psoas abscess, so the diagnosis of hematogenously disseminated tuberculosis was established. Treatment with rifampicin, isoniazid, ethambutol and pyrazinamide was started. After 6 weeks on this four-drug regimen laboratory tests were repeated. Serum creatinine had risen to $272 \mathrm{micromol} / \mathrm{l}$ with a simultaneous rise of the lymphocyte count to $1.5 \times 10^{9} / 1$. Urinalysis showed $2+$ protein, but no cells or casts. Protein excretion was $2.1 \mathrm{~g} /$ day. All four drugs were continued, albeit that the dosage of ethambutol was reduced with $50 \%$. In the ninth week of treatment a renal biopsy was performed. Light microscopy revealed normal glomeruli but the interstitium showed a dense mononuclear infiltrate, existing of lymphocytes, some plasma cells and a few eosinophils. There were no signs of acute tubular necrosis. A ZiehlNeelsen stain was negative for mycobacteria (Figure 1). On immunofluorescence: IgG was negative, IgA was positive in parts of some tubuli and IgM showed an atypical staining of the glomeruli. At the time of renal biopsy serum creatinine was $240 \mathrm{micromol} / \mathrm{l}$ and when the result of the biopsy became available it had decreased to 203 micromol/l. Because of this spontaneous recovery, all drugs were continued. Seven weeks after the renal biopsy, serum creatinine had returned to pre-treatment values. Nine months later treatment was stopped and the laboratory data were: $\mathrm{Hb} 8.0 \mathrm{mmol} / \mathrm{l}$, creatinine $88 \mathrm{micromol} / \mathrm{l}$, serum albumen $39 \mathrm{~g} / \mathrm{l}$ and protein excretion $0.2 \mathrm{~g} /$ day.

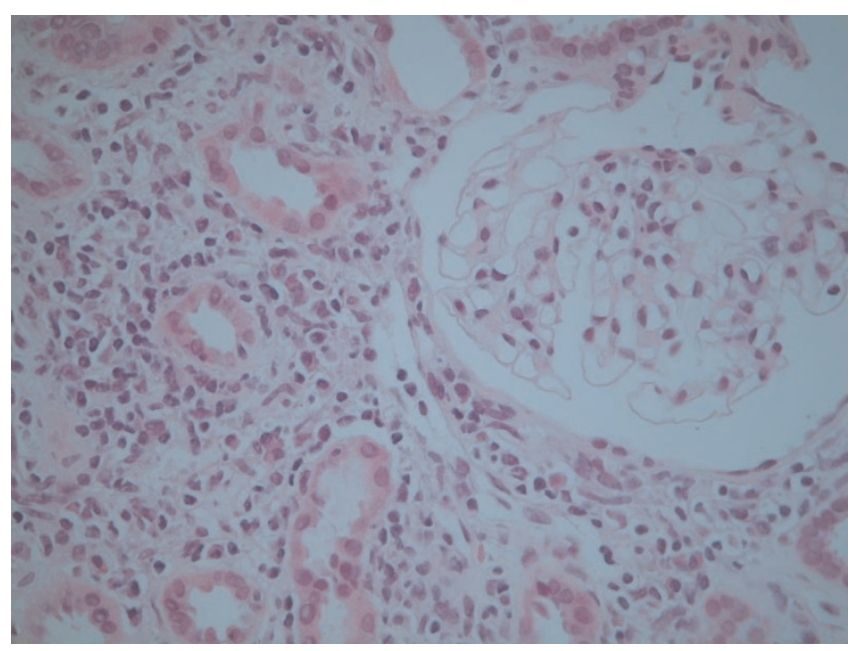

Figure I.

Renal biopsy with a dense infiltrate in the interstitium, existing of lymphocytes, some plasma cells and a few eosinophils. No signs of acid-fast bacilli or acute tubular necrosis.

\section{Discussion}

The introduction of effective anti-tuberculosis treatment has been accompanied with acute renal failure. In the early fifties of last century nephrotoxicity of streptomycin, one of the first aminoglycosides, was causative. When rifampicin became available, acute tubular necrosis was described. Almost all of the patients had rifampicin-dependent antibodies against the I-antigen which is present on erythrocytes and also on tubular epithelial cells. This I-antigen therefore could be the target through which the drug leads to acute tubular necrosis [1]. In the nineteenseventies, case histories of acute IN were published. Rifampicin was given for 21 to 71 days and renal biopsies showed interstitial infiltrates. The drug was stopped and renal function recovered. Due to this temporal relationship, an immune-mediated or direct nephrotoxicity of rifampicin was postulated [2-4]. However; such temporal relationship could also be compatible with a PR.

Paradoxical enlargement of intracranial tuberculomas during anti-tuberculosis treatment were reported in the early nineties of last century and called PR [6]. Later, with the start of highly effective anti-retroviral therapy, PR became more common in HIV-positive patients with hematogenously disseminated tuberculosis during antituberculosis treatment. Recently, even IN in such a patient has been reported. Two months after the start of the combination of anti-retroviral and anti-tuberculosis treatment, renal failure developed and renal biopsy revealed interstitial infiltrates. Assuming PR, rifabutin a rifamycin not negatively affecting the anti-retroviral therapy was continued and prednisone was added. Renal function improved within two weeks [7].

PR is defined as a transient worsening of pre-existing, often symptomless, lesions during anti-tuberculosis treatment. The etiology is unknown, although an interaction between the host's immune response and mycobacterial products has been suggested. PR occurs more frequently in HIVpositive patients. Risk factors for developing PR in HIVnegative patients are: anemia $(\mathrm{Hb}<6.8 \mathrm{mmol} / \mathrm{l})$, low serum albumin $(<30 \mathrm{~g} / \mathrm{l})$ and a low lymphocyte count $\left(<0.8 \times 10^{9} / 1\right)$. Furthermore, during PR a significant rise of the lymphocyte count $\left(>0.3 \times 10^{9} / 1\right)$ is seen [8]. Antituberculosis treatment during a PR should be continued. Our patient had hematogenously disseminated tuberculosis and abovementioned phenomena. Our hypothesis is that during the hematogenous spread mycobacteria are trapped within the kidney and cause IN i.e. PR during antituberculosis treatment. The fact that the patient received quadruple therapy for nine weeks, could explain the absence of mycobacteria in the renal biopsy.

Transient IN during rifampicin treatment in HIV-negative tuberculosis patients has been described before, but not 
recognized as PR. One patient recovered after withdrawal of pyrazinamide while rifampicin was continued. The authors postulated pyrazinamide as the cause of the nephritis [9]. Another patient developed IN while receiving triple therapy. Rifampicin and isoniazid were stopped without effect. When ethambutol was stopped as well, renal function improved. Then rifampicin and isoniazid were reintroduced without detrimental effect. Thus ethambutol was considered the culprit [10]. Both patients however, had hematogenously disseminated tuberculosis and anemia. So PR seems a more likely explanation. Unfortunately, the case histories of rifampicin-associated IN contain insufficient data to draw conclusions about a possible PR.

\section{Conclusion}

HIV-negative tuberculosis patients with well-defined risk factors may develop IN during anti-tuberculosis therapy. This nephritis should be considered a PR, thus antituberculosis drugs could be continued and corticosteroids eventually added.

\section{List of abbreviations}

IN, Interstitial nephritis; PR, paradoxical response; CT, Computed tomography; CRP, C-reactive protein.

\section{Consent}

Written informed consent was obtained from the patient for publication of this case report and accompanying images. A copy of the written consent is available for review by the Editor-in-Chief of this journal.

\section{Competing interests}

The authors declare that they have no competing interests.

\section{Authors' contributions}

JvdM reviewed the case and relevant literature and prepared the manuscript. GJ reviewed and edited the manuscript. PW performed the histological examination. All authors read and approved the final manuscript.

\section{References}

I. De Vriese AS, Robbrecht DL, Vanholder RC, Vogelaers DP, Lameire $\mathrm{NH}$ : Rifampicin-associated acute renal failure: pathophysiologic, immunologic, and clinical features. Am J Kidney Dis. 1998, 3 I:108-II5.

2. Gabow PA, Lacher JW, Neff TA: Tubulointerstitial and glomerular nephritis associated with rifampin. Report of a case. JAMA. 1976, 235:2517-2518.

3. Neugarten J, Gallo GR, Baldwin DS: Rifampin-induced nephrotic syndrome and acute interstitial nephritis. Am J Nephrol. I983, 3:38-42.

4. Power DA, Russell G, Smith FW, Simpson JG, MacLeod AM, Friend JA, Catto GR: Acute renal failure due to continuous rifampicin. Clin Nephrol. 1983, 20:155-159.

5. Yu ASL, Brenner BM. Harrison's Online Chapter 279 Tubulointerstitial Diseases of the Kidney [http://www.accessmedicine.com/ content.aspx?aid=2874673].
6. Van Bommel EFH, Stiegelis WF, Schermers HP: Paradoxical response of intracranial tuberculomas during chemotherapy: an immunologic phenomenon? Neth J Med. I99|, 38: I26-I30.

7. Jehle AW, Khann N, Sigle JP, Glatz-Krieger K, Battegay M, Steiger J, Dickenmann, Hirsch $\mathrm{HH}$ : Acute renal failure on immune reconstitution in an HIV-positive patient with miliary tuberculosis. Clin Infect Dis. 2004, 38:32-35.

8. Cheng SL, Wang HC, Yang PC: Paradoxical response during antituberculosis treatment in HIV-negative patients with pulmonary tuberculosis. Int J Tuberc Lung Dis. 2007, I I: I 290- I 295.

9. Sanwikarja S, Kauffmann RH, te Velde J, Serlie J: Tubulointerstitial nephritis associated with pyrazinamide. Neth J Med. 1989, 34:40-46.

10. Garcia-Martin F, Mampaso F, de Arriba G, Moldenhauer F, MartinEscobar E, Saiz F: Acute interstitial nephritis induced by ethambutol. Nephron. 1991, 59:679-680.

\section{Do you have a case to share?}

Submit your case report today

- Rapid peer review

- Fast publication

- PubMed indexing

- Inclusion in Cases Database

\section{Any patient, any case, can teach us something}

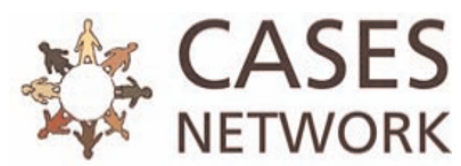

www.casesnetwork.com 